\title{
Zum Problem des Johannesevangeliums.
}

Von Wilhelm Soltau in Z abern i/Elsass.

Nachdem kürzlich das Problem des Johannesevangeliums wieder von mehreren Seiten aus in Angriff genommen ist, namentlich auch die gründlichen Forschungen Wendts ${ }^{2}$ von verheissungsvollen Anfängen wieder auf Abwege geraten sind, dürfte es vielleicht erwünscht sein, die Grundlagen, von welchen die moderne Forschung meist ausgegangen ist, einer erneuten Prüfung zu unterziehen und einiges über den Weg, der unter allen Umständen einzuschlagen ist, beizubringen.

Weiteres als einige Vorbemerkungen zur erneuten Inangriffnahme der Untersuchung können die folgenden Zeilen natürlich nicht bieten.

Pfleiderer (Urchristentum 781) fasst das Urteil der wissenschaftlichen Theologie über das vierte Evangelium kurz und bestimmt so zusammen: „Die johanneischen Reden sind so sehr aus einem Guss, Form und Inhalt so untrennbar eins, und die Reden wieder so eins mit den sie einleitenden oder illustrierenden Erzählungen, dass es unmöglich ist, das eine von dem andern zu scheiden."

Die Folgerung ist damit gegeben: das ganze Evangelium ist in allen seinen Teilen ein einheitliches Werk eines nachapostolischen Schriftstellers des zweiten Jahrhunderts, der weder Apostelschüler noch gar ein Apostel gewesen sein kann.

Von den beiden Annahmen Pfleiderers ist nun aber nur die erste richtig. Die zweite ist, wie gezeigt werden soll, falsch, und damit wird auch die Schlussfolgerung hinfällig.

Es ist nämlich bestimmt nachweisbar, dass die Redestücke ${ }^{2}$ des

- Das Johannesevangelium (Göttingen 1900)

2 Es sind dieses abgesehen von dem den Redestücken verwandten Prolog (I, $I-18) .3,13-21 ; 4,10-15 ; 4,31-38 ; 5,19-47 ; 6,32-65 ; 8,12-19,8,21-59$; 10, I-18; 10, 26-42; 14-17; dazu wohl auch $3,31^{\mathrm{b}}-36$; 12, 34-36; $44-50$ und (s. unten) $7,28-36$. 
Evangeliums nur in einem losen Zusammenhang mit demjenigen Erzählungsstoff stehen, welcher Sondereigentum des vierten Evangeliums ist, ja auch dieser ist kaum als einheitlicher $z u$ bezeichnen.

Suchen wir diesen prinzipiell abweichenden Standpunkt im Einzelnen zu begründen

Die johanneischen Redestücke sind erstlich ohne Rücksicht auf den Zusammenhang, mehrfach ohne jede Beziehung zum voraufgehenden Erzählungsstoff eingelegt.

Die Rede von der Liebe $15,9-17$ ist nicht etwa nach der Fusswaschung 13,16 eingeschoben, sondern 2 wischen die Allegorie vom Weinstock $15,1-8$ und die Rede vom Hass der Welt 15, 18-25 gestellt. - Ebensowenig ist das hohepriesterliche Gebet (I7), wie es sachlich geboten war, nach 12, 27 oder 13, 32 eingereiht. Das Bild vom guten Hirten (IO, I-I8) steht ganz unvermittelt nach den Streitworten zwischen Jesus und den Pharisäern. Das grosse Redestück 8, 12-59 ist ohne jegliche Verbindung mit der Perikope von der Ehebrecherin 8, 2-i I, oder - wenn diese unecht ist und in Wegfall kommt - auch ohne jede Beziehung zu 7, 53 geblieben. Auch die allegorische Rede ùber Jesus als "Brod des Lebens" $(6,26-65)$ stelht in keinem Zusammenhang mit dem unmittelbar Vorausgehenden. Selbst die Einleitung 1, I-I8 steht isoliert da. I,19 schliesst sich kaum äusserlich an I, 18 an; die Wiederholung von $I, 19-27$ nach 1,15 ist geradezu hart.

Aber sei es drum! Mögen auch die Reden nur ganz äusserlich eingefügt sein! Es könnte dieses vielleicht manchem conservativen Gemüt als belanglos erscheinen, ̈̈ndem ja auch zahlreiche andere Perikopen dieses und der übrigen Evangelien ohne rechten Zusammenhang aneinander gereiht sind. - Die Hochzeit von Kana ist ohne Beziehung zu der Jüngerwahl und nimmt sich sonderbar genug neben I,5I aus. Die weiteren johanneischen Erzählungen von Nikodemus, von der Samariterin, vom Aussätzigen sind zwar mit einigen motivierenden Anfangsbemerkungen versehen, stehen aber im Übrigen ohne erkennkaren Zusammenhang mit den voraufgehenden und folgenden Versen. Warum sollte es mit den Reden anders sein?

Derartigen Versuchen gegenüber muss dann zweitens darauf hingewiesen werden, dass die johanneischen Redestücke mehrfach den Zusammenhang durchbrechen, ja geradezu zerstören.

I Auch 2u 6,1-15, welches eine grosse Ideenverwandtschaft mit 6, 26f. aufweist, ist doch keine rechte Verbindung herzustellen; 6, 26 steht im Gegensatz $2 u$ 6, 14 : 
Offenbar gehören dic Angaben 10, 19f. noch zu den Ausführungen von $9^{x}$, von denen sie ganz unpassend durch 10, 1-18 geschieden werden. Das Gespräch mit Nikodemus hat bis 3, 12 seinen guten Fortgang. 3, 13 schlägt einen ganz andern Ton an und hat gar keine Bezichung zu der Unterredung selbst. Die Abschiedsrede Jesu in Joh. 14 wird 15, 26f. bez. in 16 wieder aufgenommen. Die drei Reden 15, 1-8; 15, 9-17; 15, 18-25 werden wenig passend dazwischen eingeschoben. Die allgemeinen Ausführungen $3,3 \mathrm{I}^{\mathrm{b}}$ bez. $3,32-36$ weichen auch von dem Zusammenhang von 3, 28-30 ab. Am bedenklichsten aber ist, dass die Erzählung von der Samariterin (die ursprünglich 4, I-I9; $20-25 ; 16-19 ; 27-30 ; 39-43$ umfasst hat ${ }^{2}$, durch die ganz andersartigen Redestücke 4, 10-15 und 4, 31-38 um ihren guten Zusammenhang gebracht ist. Die Rede vom Licht der Welt, 12, 44f., an das Volk: gehalten, nimmt sich doch mehr als sonderbar aus nach 12, 37 und 12,43. Selbst einige der kürzeren Herrenworte sind so zu den dem Evangelium eigentümlichen Erzählungen gesetzt worden, dass man ihren Einschub merken kann, z. B. I, 5 I lautet als erste Ankündigung an einen neugeworbenen Jünger recht befremdlich. 9, 4f. steht an der Stelle ohne rechten Sinn. Im ganzen Evangelium kehren keine Gedanken wieder, welche an den Gedankengang von I, I-I8 erinnern.

Es ist also bei der weiteren Erklärung des Evangeliums soviel festzuhalten, dass dasselbe keineswegs der „ungenähte Rock" ist, der keine Zerteilung zulässt; die Reden sind jedenfalls mehrfach ziemlich äusserlich in den Erzählungsstoff eingelegt.

Dazu kommt, dass noch ein zweiter Bestandteil des vierten Evangeliums eine besondere Herkunft hat und sich bestimmt von dem übrigen abhebt.

Voraussetzung aller weiteren Untersuchungen über das vierte Evangelium ist nämlich das feststehende Ergebnis der neutestamentlichen Forschung, dass das vierte Evangelium manche historische Berichte der synoptischen Tradition entnommen hat. Oft kommen in einem und demselben johanneischen Verse Spuren der Erzählung mehrerer synop-

I Vor.allem 10, 20-21, welche auf die Blindenheilung in 9 gehen.

2 Ohne Weiteres ist klar, dass $4,16-19 ; 4,28-30 ; 4,39-41$ Teile einer fortlaufenden Erzählung sind, welche durch 4, 20-24 und 4, 3I-38 zerrissen sind. Nun zeigt die allegorische Umdeutung des Wassertrinkens (4, Io neben 4, 7 ), dass hier Fremdartiges (4, 10-15) der Erzählung beigefügt ist, wogegen 4, 20-24 die allein passende Fortsetzung von $4, \mathrm{I}-9$ bilden würde. Es ist daher nicht nur $4,31-3^{8}$ sondern auch 4; 10-15 ein später eingeschobenes Redestück. Der Redactor brachte dann auch 4, I6-20 etwas früher, da es nach $4,25-26$. nicht mehr passend erschien. 
tischer Evangelien vor. Sogar die Zusätze und Ergänzungen des kanonischen Matthäus sind dem vierten Evangelisten bekannt (z. B. Joh. I2, I5 beruht auf Math. 21, 5). Synoptischer Herkunft sind:

I, I9-28; $31-34$, vielleicht auch manches in $35-42$;

2; $13-17 ; 19 ; 22$; $^{x}$

4, 43-54;

$6, \mathrm{I}-25$; .

$6,66-71$

9, $1 ; 6-23$;

II, 47-55 (57);

I2, 3-8; 12-16;

13, 26-27;

18 ,

I9, I-24; $38-42$;

20, I-2; II $-13 ; 19-23$.

Dazu kommen vereinzelte Sprüche und Erzählungsmomente, wie in: 2,$19 ; 4,44 ; 5,8 ; 12,25,27,40 ; 13,16,20-21,38 ; 14,13 ; 15$, 20; 16, 32; 18, II.

Als der dem vierten Evangelisten eigentümliche Erzählungsstoff bleiben somit nur folgende Abschnitte übrig:

I. I, (35-42) 43-5I Jüngerwahl, wozu wahrscheinlich auch der aus I, 35 anticipierte Gedanke I, 29 kommt („Lamm Gottes“)

2. 2, I-II Hochzeit zu Kana.

3. 3, I-12 Nikodemus.

4. 3, 22-3I a Johannes und Jesus.

5. 4, I-9; 20-30; 39-42; (4, I6-I9?) die Samariterin.

6. 5, I-I6 (I8) der Kranke $2 \mathrm{u}$ Bethesda.

7. 7, I-8, I Jesus am Laubhüttenfest ${ }^{2}$.

8. 8, 2-I I die Ehebrecherin 3 .

9. 9, 23-4I Blindenheilung 4 .

10. 12, 20-33 Jesu Worte an Philippus und Andreas (vielleicht noch weiter $12,37-43$ ).

I Dieser Erzāhlungsstoff ist aber überall mit originellen johanneischen MLotiven durchsetzt.

2 Vielleicht sind in C. 7 einige kleinere Einlagen des Evangelisten, so namentlich $7,28-30$ und $7,33-36$ bez. das ganze Stück $7,28-36$.

3 Doch fehlt dieselbe in den alteren Handschriften und weicht rom sonstigen Sprachgebrauch des Evangeliums ab.

49,$1 ; 6-23$ ist aus synoptischen Elementen zusammengesetzt; auch 9, 2-3 ist vielleicht Reminiscenz aus Ic 13,2 . Einiges in 9,18 f. ist schon aus 9,23 f. entnommen. 
12. 13, 2-15 (16-20) Fusswaschung (und vielleicht dazu auch 13 , $3 \mathrm{r}-36)$ ?

13. 19, 25-37 selbständige Zusätze bei der Kreuzigung.

14. 20, 14-18; 25-29 dic aussersynoptischen Erscheinungen Jesu.

Ausserdem ist noch die ganz singuläre Lazaruserzählung (Joh. II) zu nennen, über welche zum Schluss gesondert gehandelt werden soll.

Nachdem hier dargelegt ist, dass das viertc Evangelium aus drei verschiedenartigen Bestandteilen besteht, ist der Frage näher zu treten, wie man sich die Verbindung derselben durch den Evangelisten zu denken habe.

Ist bei der grossen Verschiedenartigkeit der Teile die Annahme einer einheitlichen Bearbeitung festzuhalten?

Das hier klargelegte Verhältnis von Erzählungsstoff und Redestücken könnte unter andern Umständen die Verınutung nahe legen, dass die Reden erst später, ziemlich äusserlich, von einem Ergänzer eingeschoben seien.

Diese Annahme ist aber a limine abzuweisen; denn abgeseher von der sprachlichen Einheit des Evangeliums, abgesehen davon, dass ja gerade von dem geistigen Urheber der Redestuicke manche kleinere Einlagen herrühren und Correcturen an der Erzählung vorgenommen sind '2, besteht trotz aller Gegensätze zwischen johanneischen Reden und johanneischer Erzählung doch auch eine gewisse Verbindung zwischen Reden und Erzählungsstoff. Diese Verbindung, welche in stilistischer und logischer Weise oft zu wünschen übrig lässt, besteht trotzdem in stofflicher, in inhaltlicher Beziehung.

Vereinzelt sind zwar einige Spriiche aus den Synoptikern in die johanneischen Reden miteingeschoben. An keiner Stelle aber bietet irgend ein Gedanke der Synoptiker das Thema jener Reden." Das Umgekehrte ist hinsichtlich des johanneischen Erzählungsstoffes zu constatieren. Trotz aller Eigenartigkeit der johanneischen Reden beruhen sie doch in einigen ihrer Hauptgedanken auf jener johanneischen Erzählungsquelle, was nicht ausschliesst, dass sie dieselben in vielfacher Hinsicht weiter gebildet und umgestaltet haben.

Folgende Sprüche aus dem Erzählungsbericht bieten einige verwandte Ideen, ja bilden zuweilen sogar das Thema zu den Reden selbst.

I I3, 34 ist sachlich' gleich Mc I2, 3I, aber sowohl die Form von 13, 33, wie der Zusatz 13, 36 lassen auf eine originale Herkunft schliessen.

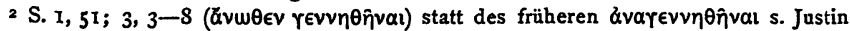
Apol. 1, 61.

22. 5. 1901 . 
Der Gedanke von

$7,7^{x} \quad$ ist verwandt worden in der Rede

I $5,18-25$.

$7,16-18$

13,33

"

" $"$
" " , "

" $, \quad, \mathrm{I}$ $16,16 f$

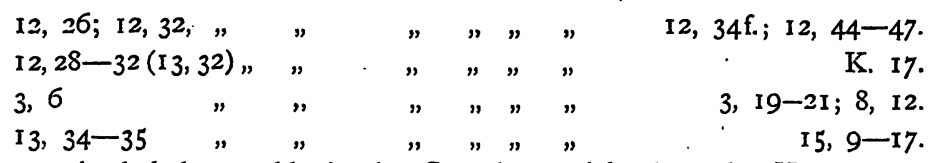

Auch haben wohl eirzelne Sprüche, welche jetzt das Hauptthema der grossen Reden bilden, schon in der Quelle gestanden. So I0, I2 (für IO, I-I8); I5, I-2 (für I 5, I-8); 16, I9-22 (für I6).

Danach ist die Folgerung unabweisbar: Ein bereits vorliegender Erzählungsstoff ist durch den später schreibenden Evangelisten mit erbaulichen Reden ausgestattet worden. Der Erzählungsstoff besass für den Evangelisten bereits ein solches Ansehen, dass er ihn nicht allzu frei umzugestalten wagte, sondern ihn nur zu vervollständigen suchte. ${ }^{2}$ Und andererseits werden auch wohl einige Reden an sich schon einen solchen Wert in den Augen des Bearbeiters dieses Erzählungsstoffes besessen haben, dass er sie nicht ohne Not zerteilen wollte. Der Erzählungsstoff muss demnach jedenfalls bereits schriftlich fixiert dem Evangelisten vorgelegen haben, wahrscheinlich aber waren auch schon "einige der Reden, bevor sie in das Evangelium eingelegt wurden, von ihm aufgezeichnet gewesen.

Anders verfährt der Evangelist mit den synoptischen Berichten. Die letzteren sind anerkanntermassen meist gedächtnismässig in seine Darstellung aufgenommen. Vielfach folgt der Evangelist sogar einer traditionellen Weiterbildung des synoptischen Materials.

Welcher Art war diese dem vierten Evangelisten eigentümliche Erzählungsquelle? Abgesehen von den legendarischen Ergänzungen zur

I Natürlich mit Berücksichtigung des Unterschiedès zwischen Jesu Brüdern 7, 5 und Jesu Jüngern $15,19$.

2 Eine gewisse Analogie zum Matthäusevangelium liegt vor. Wie ein Protomatthäus (vgl. Soltau: Eine Lücke der synoptischen Forschung, S. 14f.) seine Logiaquelle durch Marcusberichte zu einer Geschichtserzählung umwandelte und dabei die Logia zu grösseren Reden verband, so hat auch der vierte Evangelist seine Quelle durch synoptische Berichte und durch Reden erweitert. Noch deutlicher aber tritt bei dem Vergleich die Verschiedenheit in der Arbeitsweise beider Evangelisten hervor; der erste Evangelist hielt sich treu an die Erzählung des Marcus und brachte in die Logia trotz aller rhetorischen Erweiterung nur wenjg Eigenartiges hinein. Der vierte Evangelist behandelte beide Stoffe ganz frei.

Zeitschrift f. d. neutest. Wiss. Jahrg. II. 190r. 
Auferstehungsgeschichte $(20,14-18 ; 25-29)$, die vielleicht nicht einmal in der Quelle selbst standen, sondern als freie Weiterbildungen der synoptischen Berichte' gelten dürfen, sind alle $14 \mathrm{Abschnitte}^{2}$ des Erzählungsstoffes derart, dass sie nicht einen fortlaufenden historischen Bericht über dic Thaten Jesu bilden, sondern vielmehr nur eine Sammlung von Aussprüchen Jesu mit historischer Einführung enthalten. Es sind Logia $(\Lambda \mathrm{J})$.

Durch diese Erkenntnis fällt ein helles Licht auf die ganze Beschaffenheit der Nachrichten dieser Quelle $(\Lambda J)$. Eine chronologische Anordnung lag ihr völlig fern, ein chronologisches System darf also auch nicht in ihr vermutet werden. Wenn z. B. die Tempelaustreibung an den Anfang von Jesu Thätigkeit gesetzt wird $(2,15 f$.), so beruht das nicht auf einer anderweitigen historischen Berichterstattung, sondern auf dem zufälligen Umstand, dass einige Worte Jesu (z. B. 2, 19) und andere mit ihnen zusammenhängende Erzählungen über Jesu Thätigkeit im Tempel mehr zu Anfang der Redequelle standen. Bei einer solchen Logiaquelle $(\Lambda J)$ ist es auch wohl verständlich, wenn hier und da einzelne echte Reminiscenzen localer Art miterwähnt werden, 3 ohne dass doch im übrigen eine .klare Übersicht über die Örtlichkeiten, an welchen Jesus thätig war, vorhanden zu sein brauchte.

Es ist hier nicht der Ort, diesen auch von anderen schon bemerkten Gegensatz, welcher zwischen den Johannesperikopen und den Redestuicken besteht, im einzelnen auszuführen. Gerade der Abschnitt in Wendt's Johannesevangelium, welcher von den "Anzeichen des Benutztseins einer Quellenschrift im vierten Evangelium" handelt (S. 45-100) hat so manche treffende Beobachtung nach dieser Richtung gemacht, dass er mich einer weiteren Erörterung desselben hier überhebt. Besonders charakteristisch sind ja die doppelten Erklärungsversuche, bald mehr platterer Art, bald "pneumatischer". So z. B. 12, 32-33 und daneben 12, 34-36, oder 4, 10-14 neben 4, 9, oder 7, 39 neben 4, 10-15; 6, 35. Weiteres vgl. in meinem Buch „Unsere Evangelien, ihre Quellen und ihr Quellenwert (Leipzig ' I90I), S. II4f.

I S. H. Holtzmann, Handkommentar IV, S. 201.

2 Auch die Hochzeit von Kana, wenn eben beachtet wird, dass sie ihre eigentliche Deutung erst bei einer allegorischen Erklärung erbält.

3 Die Erzählung berührt den Teich Siloah 9, 7, die Halle des Salomo im Tempel 10, 23, den Kidronbach 18, 1 , den Palast des Hannas 18 , 15 , das Prätorium IS, 28 , Golgatha 19, 17. s. O. Holtzmann, Das Johannesevangelium, S. 189; Soltau, Unsere Evangelien, ihre Quellen und ihr Quellenwert (Leipzig 1901), S. II3f. 
Mit der Constatierung einer solchen Logiaquelle, welche bedeutend älter als das Evangelium war, ist es aber allein nicht gethan. Wichtiger ist, dass ihre Glaubwürdigkeit und ihre Herkunft im einzelnen definiert wird.

$\mathrm{Da}$ wird es denn jedem Einsichtigen klar sein, dass der Inhalt dieser Quelle Dinge von sehr verśchiedenem Wert enthält.

Einzelne Sprüche tragen den Stempel: der Echtheit, der tiefsten und innigsten Frömmigkeit an sich. So Jesu Wort über die Liebe 13, 33-35, das Wort vom guten Hirten (10, 12), die Allegorien vom Weinstock 15, 2-5, vom Brod des Lebens 6, 35. Auch die Grundzüge der Gespräche mit Nikodemus, mit der Samariterin, oder das Wort an Petrus 13, 36 sind wahrscheinlich echt, auf alle Fälle aus älterer Tradition. Manches andere erinnert an die dürtigeren Wunderberichte des Marcus, so 5, If.; 9, 23 f., und verbürgt vielleicht gerade damit eine gewisse Ursprünglichkeit der Berichterstattung, die allerdings von dem, der sie verzeichnet hat, mit einigen allegorischen Bestandteilen ausgestattet sein müsste. Dagegen ist z. B. die Bedeutung der Perikope 2, If. und manches Allegorische ${ }^{2}$ im Gespräch mit der Samariterin in der jetzigen Berichterstattung völlig verblasst. Und nun gar manche der historisch-seinwollenden Sonderberichte ( $1,44 f ., 9,35 \mathrm{f}$., 19, 3I-37) machen einen durchaus legendenhaften Eindruck. Endlich ist zu beachten, dass fast überall höchstens von späteren apostolischen Erinnerungen über Jesu Person; nicht von einer Authentie der Worte Jesu geredet werden darf.

Der Nachweis, dass eine besondere Logiasammlung dem vierten Evangelium 2u Grunde lag, führt auch auf die Lösung des Hauptproblems, wie es möglich war, dass die gesammte kirchliche Tradition den Apostel Johannes als Verfasser dieses Evangeliums hinstellen, und speziell 13,23; 19, 26-27; 20, 2 deutlich. genug auf ihn als Gewährsmann hinweisen konnte. Namentlich auch das bestimmte Zeugnis des Ergänzers 21, 24 liess ja von jeher keine andere Alternative $\mathrm{zu}$, als apostolischen Ursprung oder tendenziösen Betrug.

Diese grosse Schwierigkeit ist durch den Nachweis einer Quelle des Johannesevangeliums beseitigt, wenn anders es glaubhaft gemacht werden kann, dass diese Logiasammlung in irgend einer Weise auf den Apostel Johannes zurückgeführt werden kann.

Von vornherein ist dabei allerdings weniger an eine schriftstellerische Thätigkeit des Apostels Johannes zu denken. Vielmehr wăre anzu-

1 Man denke dabei an verwandte Gedanken der Synoptiker wie 2. B. Mt 11, 28-30.

2 So weisen die fünf Männer der Samariterin auf die fünf Stämme der Samariter hin. 
nehmen, dass Aufzciclinungen anderer nach früheren mündlichen $\mathrm{Be}$ richten des Apostels stattgefunden haben.

In dieser Einschränkung des Urteils aber lassen sich für den apostolischen Ursprung dieser Logiasammlung manche gewichtige Gründe beibringen. Vor allem: Sämtliche Sonderberichte der Leidenserzählung fuhren auf johanneischen Ursprung: sowohl das letzte Wort Jesu an seine Mutter und an Johanncs (19, 26-27), wie die Erzäklung über den Lanzenstich I9, 3I-37 (vgl. dazu Apc I, 7) und die besondere Berücksichtigung $(20,3$ f.) des anderen Jüngers, der Petrus zuvorkam, d. i. des Johannes.

Auch die zahlreichen besonderen Ortsangaben und Hinweise auf das jüdische Leben in Palästina führen auf einen Berichterstatter hin, der zur Zeit Jesu selbst in Palästina anwesend gewesen ist.: Johannes gilt der kirchlichen Tradition zufolge als Verkündiger der Liebe. „Kindlein, liebet euch" soll nach Polykarp die stete Mahnung des greisen Apostels gewesen sein. 13, 33-35 bietet die gleiche Angabe, sogar in ähnlicher Fassung.

Jedenfalls ist auf diesem Wege die Möglichkeit gegeben, den apostolischen Ursprung der Grundschrift beizubehalten, ohne im übrigen den späten Abschluss des ganzen Evangeliums durch den Presbyter, welcher den I. Johannesbrief geschrieben hat, abzuleugnen. I, I4 ist nicht von einem persönlichen Schauen des Evangelisten zu deuten, sondern betont ganz allgemein, dass wir Menschen, die Menschheit ein solches Wunder, dass der Logos Fleisch geworden sei, geschaut haben. ${ }^{2}$

Nur eine Schwierigkeit vermag ich nicht völlig zu erklären: „Die singuläre Lazarusepisode." Dieselbe weist mit II, 2 merkwürdiger Weise schon auf das erst 12, 3 zu Erzählende hin, setzt das I2, 3 f. Erzählte schon voraus. Damit giebt auch sie sich als eine spätere Einlage zu erkennen. Wahrscheinlich ist daher, dass $\mathrm{I} I, \mathrm{I}-46$ bez. $\mathrm{I}_{2}, \mathrm{I}^{\mathrm{b}}$ erst von dem Evangelisten, der zugleich seine Reden einschob, eingefügt ist.

Fassen wir die Ergebnisse noch einmal hurz zusammen. Es gab eine teils von Berichten des Apostels Johannes, teils von Angaben aus dem Kreise seiner Zuhörer abstammende Sammlung von Perikopen, welche Gespräche Jesu mit kurzen Einführungen enthielt.

I Vgl. O. Holtzmann a. a. O. S. 191 und H. Holtzmann, Hand-Commentar, TV, S. 65.

2 Die Nachbildung dieser Worte im I. Johannesbrief I, 1-3 wird schon durch I, 5 auf das rịchtige Mass d. h. geistigen Schauens zurückgeführt. Vielleicht ist I Joh $\mathrm{I}-5$ übrigens älter als Joh $\mathrm{I}, \mathrm{I}-\mathrm{I} 8$. 
Ihr Wert war ungleichartig. Manches ging auf wirkliche Erinnerungen des Apostels zurück. Einige Allegorien und Aussprüche Jesu verraten die Ursprünglichkeit. Anderes war secundärer Natur. Diese Sammlung hatte schon beim Tode des Apostels Johannes ein gewisses kanonisches Ansehen. Dieselbe wurde, sei es allmählich, ${ }^{\circledR}$ sei es auf einmal, als sich der Wunsch Bahn brach, auf Grund der johanneischen Logiasammlung ein zusammenhängendes Lebensbild Jesu zu erhalten, durch synoptische Berichte ergänzt. Daneben waren, im Anschluss an einige der tiefsten Aussprüche der Johanneslogia, Reden der kleinasiatischen Presbyter entstanden, welche einzelne jener Logia weiterausspinnend die philosophische und mystische Ideenwelt dieser $\mathrm{Kreisè}^{2}$ widerspiegelten.

Einer derselben, vermutlich der Presbyter Johannes, der Verfasser des I. Johannesbriefes, unternahm es, diese Reden über Jesu Person, in deren Anschauungen ihm das Wesentliche des Christentums zu liegen schien, zu sammeln und dieselben dann in die schon bestehende Sammlung von $\Lambda \mathrm{Joh}$. einzuschieben; zugleich nahm er dabei eine Überarbeitung des gesammelten Stoffes vor. So wurde äusserlich eine Einheit, namentlich auch in sprachlicher Hinsicht, hergestellt. Doch hinderte das kanonische Ansehen, in welchem bereits die Johanneslogia zu seiner Zeit standen, den Evangelisten grössere Umarbeitungen vorzunehmen. Zum Teil liess er die Redestoffe bei einander, wie I, I-I8; 8, 12-59; IO, 1-18 bez. 26-42; 14-17. An anderen Stellen durchbrach er bei ihrer Einlage den früheren Zusammenhang (wie in I3, 34 und 4, 10). Er ging nicht soweit, alle Incongruenzen zwischen der Quellenschrift und seinen Reden zu tilgen. Der Nachweis, dass und wo solche existieren, sowie die Thatsache, dass die Reden äusserlich eingelegt sind, zeigt aufs klarste, dass von einer völligen Einheitlichkeit des Evangeliums keine Rede sein kann. Die Ünterscheidung von Johanneslogia und den Ausführungen des Evangelisten giebt den Schlüssel zur Lösung des Johannesproblems im einzelnen.

I Jedenfalls früh sind 2,13 f. und 4, 46-54 eingelegt.

2 Das ist der richtige Kern jener verwegenen Vermutungen, welche Kreyenbühl in seinem „Evangelium der Wahrheit" (Berlin, 1900) I, 145 f. geäussert hat. Das vierte Evangelium ist mit nichten „ein schriftstellerisches Produkt der bäretischen Gnosis.“ 